\title{
Monoidal Controlled Poincaré Duality
}

\author{
René dePont Christensen \\ Hans J. Munkholm*
}

September 16, 2002

\begin{abstract}
We define cochains for controlled spaces, and set up Poincaré duality for the universal cover of an orientable, controlled manifold. The map from the controlled cochains to the controlled chains is a suitably defined cap product with a (non controlled) locally finite, singular orientation cycle of the manifold.

The type of control used, monoidal control over a fixed space $Z$ (with a given "monoidal control structure"), is inspired by the entourage viewpoint of Higson, Pedersen and Roe, [8]. It includes as special cases the bounded control and the continuous control of Anderson and Munkholm, [1] and [2]. The cochains and the duality result were used in Attie's study of manifolds of bounded geometry, apparently without ever being defined, [3].
\end{abstract}

Mathematics Subject Classification 2000

Primary: 57N65, 57P10, 55M10

Secondary: 18A25, 18E10, 55U15, 55U30

*Supported in part by SNF, Denmark, under grant no. 9802057 


\section{Contents}

1 Introduction 3

2 Topology with Monoidal Control 4

3 Categories with a Monoid Action 5

4 A Reduction to Cyclic Monoids $\quad 8$

5 The Universal Cover of a Controlled Space 9

6 Controlled Chains and Homology 10

$7 \quad N$-small chains and locally finite chains $\quad 11$

8 Controlled cochains and cohomology, "upstairs" 13

9 The controlled cap product $\quad 16$

10 Controlled Poincaré Duality, "upstairs" 17

11 Appendix $\quad 18$

11.1 Chain "groups" are projective . . . . . . . . . . . . . . . . 18

11.2 Remarks on Poincaré duality "downstairs" . . . . . . . . . . . . . . . . . . 19

11.3 Remarks on the cellular (or simplicial) case . . . . . . . . . . . . . . . . . . 19 


\section{Introduction}

In [1], Anderson and the second author of the present paper set up basic parts of algebraic topology in the context of spaces controlled over a fixed space $Z$. Here, a space controlled over $Z$ is simply a pair $(E, p)$ with $p: E \rightarrow Z$ a continuous map of topological spaces. Moreover, $Z$ comes with some extra structure, the role of which is to restrict the set of continuous maps $E_{1} \rightarrow E_{2}$ that are allowed as controlled maps $\left(E_{1}, p_{1}\right) \rightarrow\left(E_{2}, p_{2}\right)$. In [1], the extra structure on $Z$ is a so called bounded control structure, and the maps allowed are called $b c$ which is short for boundedly controlled. The setup of [1] includes a Whitehead Theorem which characterizes a controlled homotopy equivalence $f:\left(E_{1}, p_{1}\right) \rightarrow\left(E_{2}, p_{2}\right)$ as a controlled map which induces an isomorphism on all homotopy "groups". Also included is a Hurewicz Theorem that allows one to compute certain homotopy "groups" as homology "groups" of "universal covers". Here the homology "groups" $H_{n}(\widetilde{(E, p)})($ for $n \geq 0)$ and the homotopy "groups" $\pi_{n}(E, p)$ (for $n \geq 2$ ) are not really groups, but objects in an abelian category which is canonically associated to the controlled space $(E, p)$, and which is analogous to the category of $\mathbb{Z}\left[\pi_{1}\left(E, e_{0}\right)\right]$-modules in the non-controlled case (as usual $\pi_{0}$ and $\pi_{1}$ are special cases). Moreover, the homology "groups" are obtained by taking homology of chain "groups" in the above mentioned abelian category.

In [3], Attie describes how such a bc approach to algebraic topology can be applied to study manifolds and simplical complexes of bounded geometry (= bg for short). It is his goal to set up a surgery theory in the $b g$ context, and in particular he gives the following definition:

Definition 5.1 ( [3], p. 521) A Poincaré duality space $Y$ of bounded geometry over a simplicial complex $X$ of bounded geometry is defined to be a simplicial complex of bounded geometry over $X$ so that there is a fundamental class in the top dimensional controlled homology of $Y$ so that taking the cap product $-\cap[y]$ induces a simple homotopy equivalence of bounded geometry between controlled chains and controlled cochains of $Y$.

This definition may seem innocuous enough. However, the type of controlled homology "groups" defined in [1], do not have elements, so the fundamental class [y] does not immediately have any home to live in. In addition, in [1] there is absolutely no mention of cohomology or cochains, and in [3] no other reference for such matters is given, so the target of the Poincaré duality map also seems to be missing. In the present paper we set up a definition of cochains/cohomology, and we prove the expected Poincaré Duality Theorem for manifolds in the controlled setting. Along the way it turns out that the proper home for the fundamental class is not a controlled "group" at all. It is simply the (classical, uncontrolled) locally finite, top dimensional, homology group of the manifold.

Actually, here we work with a more general type of control, called monoidal control (= $m c$ ). In addition to the bounded control of [1], and hence the $b g$ control of [3], monoidal control also includes a version of continuous control, [2], as a special case. Basic material concerning monoidal control is exposed in [5] but below we do give a review including precise references to [5]. 
The present paper, as well as [5], report on the first named author's Ph.D. thesis, [4], written with the second author as advisor.

Acknowledgement We want to thank Stratos Prassidis for pointing out to us that Dold's treatment of Poincaré Duality, in [6], fits better into the present picture than the one we originally had in mind. Also our thanks go to Douglas R. Anderson and Jesper M. Møller for valuable comments given when they served on the junior author's Ph.D. committee.

\section{Topology with Monoidal Control}

Let $Z$ be a topological space. As usual, a space controlled over $Z$ is simply a pair $(E, p)$ consisting of a topological space $E$ and a continuous ${ }^{1}$ map $p: E \rightarrow Z$. To describe the notion of a controlled map $f:\left(E_{1}, p_{1}\right) \rightarrow\left(E_{2}, p_{2}\right)$, a "monoidal control structure" has to be given on the space $Z$. We proceed to describe what this means. Details can be found in the first named author's thesis, [4] or in our joint paper, [5].

Let $\Delta(Z)$ denote the diagonal in $Z \times Z$ and consider the set

$$
\mathcal{O}(Z)=\{N \subseteq Z \times Z \mid N=\Delta(Z) \text { or } N \text { is an open neighborhood of } \Delta(Z)\} .
$$

The elements of $\mathcal{O}(Z)$ are viewed as (reflexive) relations from the set $Z$ to itself. As such they admit a composition, written simply as concatenation, so $\mathcal{O}(Z)$ is a monoid with $u=\Delta(Z)$ as a unit. Moreover, $\mathcal{O}(Z)$ is also a poset, under $\subseteq$, and the composition map $(N, M) \mapsto N M$ respects the partial order in both variables. We may summarize this by saying that $\mathcal{O}(Z)$ is a monoid in the category of posets, having $u$ as a unit as well as a minimal element.

We also consider the poset $\mathcal{P}(Z)$ of subsets of $Z$ partially ordered by $\subseteq$. If $N \in \mathcal{O}(Z)$, and $K \in \mathcal{P}(Z)$, we write

$$
N(K)=\{z \in Z \mid \exists x \in K:(x, z) \in N\}
$$

for the image of $K$ under the relation $N$. This defines an action of the monoid on the poset with everything taking place in the category of posets.

Definition 2.1 (Definitions 6.4 and 6.1, [5]) A monoidal control structure on $Z$ is a pair $(P, \mathcal{M})$ with the following properties

(i) $\mathcal{M}$ is a submonoid of $\mathcal{O}(Z)$. Moreover, $u \in \mathcal{M}$ and $\mathcal{M}$ is closed under inversion of relations, $M \mapsto M^{-1}$.

(ii) $P$ is a sub-poset of $\mathcal{P}(Z)$, containing all one point subsets of $Z$, and not containing the empty set.

\footnotetext{
${ }^{1}$ Some authors, e.g. Pedersen, Higson and Roe, [8], do not require continuity, but for the present setup it is needed.
} 
(iii) The action of $\mathcal{O}(Z)$ on $\mathcal{P}(Z)$ restricts to an action of the monoid $\mathcal{M}$ on the poset $P$.

(iv) If $K \subseteq Z$ is relatively compact, then $M(K)$ is relatively compact for each $M \in \mathcal{M}$.

(v) $\bigcup_{M \in \mathcal{M}} M=Z \times Z$.

The triple $(Z, P, \mathcal{M})$ will be called a monoidal control space.

When the poset $P$ is considered as a category in the usual fashion, we write it as $\mathcal{P}$.

When $Z$ has a preferred monoidal control structure, and $\left(E_{i}, p_{i}\right), i=1,2$, are spaces controlled over $Z$, a monoidally controlled $\left(=m c\right.$, for short) map $f:\left(E_{1}, p_{1}\right) \rightarrow\left(E_{2}, p_{2}\right)$ is defined to be a continuous map $f: E_{1} \rightarrow E_{2}$ for which there exists an $M \in \mathcal{M}$ such that $p_{2}(f(e)) \in M\left(\left\{p_{1}(e)\right\}\right)$ for each $e \in E_{1}$. We think of this as saying that $f$ respects the reference maps $p_{1}, p_{2}$ up to a distortion bounded by the relation $M$.

Such maps are the morphisms of the category $\mathcal{T} \mathcal{O} \mathcal{P}^{\mathcal{M}} / Z$ of spaces controlled (by $\mathcal{M}$ ) over $Z$, or more correctly/pedantically, over $(Z, P, \mathcal{M}) .{ }^{2}$ In this category, one immediately has many of the features, problems, and solutions known from standard topology. In particular, one can do mc homotopy theory after defining an mc homotopy to be an mc map with domain of the form $(E, p) \times I:=\left(E \times I, p \circ \operatorname{proj}_{1}\right)$. An $m c C W$ complex is an object $(E, p)$ where $E$ has a given finite dimensional $\mathrm{CW}$ decomposition with cells that are uniformly bounded, in the sense that there exists an $M \in \mathcal{M}$ (depending on $E$, of course) such that any cell $\sigma$ of $E$ has $p(\sigma) \subseteq M(\{z\})$ for some $z \in Z$. On the other hand, an mc manifold is simply an object $(M, p)$ where $M$ happens to be a manifold; it should be considered mc compact if the map $p$ is proper.

All of this is described in more detail in [5] to which we also refer for examples and for the fact that the worlds of bounded control, as in [1], or continuous control, as in [2], do fit nicely into the present setup.

\section{Categories with a Monoid Action}

We recall the basic algebra and category theory as it is set up in [4] and/or [5]. Let $\mathcal{M}$ be any monoid in the category of posets, and assume as before that $u \in \mathcal{M}$ is simultaneously a unit and a minimal element. Moreover, let $\alpha: \mathcal{M} \times \mathcal{C} \rightarrow \mathcal{C}$ be an action of $\mathcal{M}$ on some category $\mathcal{C}$. There results a collection of morphisms in $\mathcal{C}$

$$
\Sigma_{\mathcal{M}}=\left\{\alpha\left(u \leq M, i d_{C}\right): C \rightarrow M(C) \mid M \in \mathcal{M}, C \in \mathcal{C}\right\},
$$

where $M(C):=\alpha(M, C)$. In section 3 of [5], it is shown that this collection admits a calculus of left fractions in the sense of [11]. Therefore (compare Lemma 1.5, p. 6 of [1], if need be), the category of fractions $\mathcal{C}\left(\Sigma_{\mathcal{M}}^{-1}\right)$ and the corresponding universal functor $Q_{\mathcal{M}}: \mathcal{C} \rightarrow \mathcal{C}\left(\Sigma_{\mathcal{M}}^{-1}\right)$ admit a very concrete interpretation:

\footnotetext{
${ }^{2}$ Note that the poset $P$ does not really enter into the description of the topological side of our world. It will become necessary when we set up the algebra in the next session.
} 


\section{Proposition $3.1 \quad$ (i) The objects of $\mathcal{C}\left(\Sigma_{\mathcal{M}}^{-1}\right)$ are those of $\mathcal{C}$.}

(ii) Each morphism $f \in \mathcal{C}\left(\Sigma_{\mathcal{M}}^{-1}\right)\left(C_{1}, C_{2}\right)$ is represented by a morphism $f_{M} \in \mathcal{C}\left(C_{1}, M\left(C_{2}\right)\right)$ for some $M \in \mathcal{M}$. Another morphism, say $f_{N} \in \mathcal{C}\left(C_{1}, N\left(C_{2}\right)\right)$, represents the same morphism $f$ if and only if there is an $L \geq M, N$, such that $\alpha\left(M \leq L, i d_{C_{2}}\right) \circ f_{M}=$ $\alpha\left(N \leq L, i d_{C_{2}}\right) \circ f_{N}$.

(iii) The composition of $f$ with a morphism $g$, represented by $g_{K} \in \mathcal{C}\left(C_{2}, K\left(C_{3}\right)\right)$, is represented by

$$
(g \circ f)_{K M}:=\alpha\left(M \leq K M, g_{K}\right) \circ f_{M} \in \mathcal{C}\left(C_{1}, K M\left(C_{3}\right)\right) .
$$

(iv) Finally, for any $h \in \mathcal{C}\left(C_{1}, C_{2}\right), Q_{\mathcal{M}}(h)$ is the morphism represented by $h_{u}:=h$.

In our applications, the category $\mathcal{C}$ will be a functor category, say $\mathcal{A}^{\mathcal{B}}$, and the (right) $\mathcal{M}$ action will be inherited from a given (left) action of $\mathcal{M}$ on the domain category $\mathcal{B}$. We proceed to describe the values of the variables that are relevant for the present paper.

The monoid variable, $\mathcal{M}$, comes from a monoidal control space $(Z, P, \mathcal{M})$ as defined in the preceding section.

The target variable, $\mathcal{A}$, will be the category, $\mathcal{A B}$, of abelian groups, the category, $\mathcal{T O} \mathcal{P}$, of topological spaces, or the category, $\mathcal{C W}$, of $\mathrm{CW}$ complexes.

Finally, we need two types of values for the source category. One is $\mathcal{B}=\mathcal{P}$, i.e., the poset $P$ from the given control space, viewed as a category. The other type arises from a given object $(E, p) \in \mathcal{T} \mathcal{O} \mathcal{P}^{\mathcal{M}} / Z$. It is defined to be the wreath product construction (in the category of categories)

$$
\mathcal{B}=\mathcal{P} \mathcal{G}_{1}(E, p):=\mathcal{P} \int \mathcal{G}_{1}(E, p),
$$

where $\mathcal{G}_{1}(E, p): \mathcal{P} \rightarrow \mathcal{G P O I D}$ is the functor that associates to any $K \in P$ the fundamental groupoid of the subspace $p^{-1} K \subseteq E$. We recall this construction: An object of $\mathcal{P G}_{1}(E, p)$ is pair $(K, e)$ with $K \in P$ and $e \in p^{-1} K \subseteq E$. A morphism $(i, \omega):(K, e) \rightarrow\left(K^{\prime}, e^{\prime}\right)$ consists of an inclusion $i: K \rightarrow K^{\prime}$ in the category $\mathcal{P}$, and a homotopy class of paths in the (bigger) space $p^{-1} K^{\prime}$ from $e^{\prime}$ to $e$. The composition in $\mathcal{P} \mathcal{G}_{1}(E, p)$ is defined in an obvious way, and the action of the monoid $\mathcal{M}$ on $\mathcal{P} \mathcal{G}_{1}(E, p)$ is given by the $\mathcal{M}$ action on the first variable $K$ from $P$.

Proposition 3.2 For $\mathcal{A}=\mathcal{A B}$, the categories $\mathcal{A B}^{\mathcal{P}}\left(\Sigma_{\mathcal{M}}^{-1}\right)$ and $\mathcal{A}^{\mathcal{P G}}(E, p)\left(\Sigma_{\mathcal{M}}^{-1}\right)$ are abelian and have enough projectives.

For any category $\mathcal{A}$, any mc map $f:(E, p) \rightarrow\left(E^{\prime}, p^{\prime}\right)$ induces a functor

$$
f^{!}: \mathcal{A}^{\mathcal{P} \mathcal{G}_{1}\left(E^{\prime}, p^{\prime}\right)}\left(\Sigma_{\mathcal{M}}^{-1}\right) \rightarrow \mathcal{A}^{\mathcal{P} \mathcal{G}_{1}(E, p)}\left(\Sigma_{\mathcal{M}}^{-1}\right)
$$

which is canonical up to a natural equivalence. For $\mathcal{A}=\mathcal{A B}$, this is an exact functor. 
If an me map $f:(E, p) \rightarrow\left(E^{\prime}, p^{\prime}\right)$ is controlled by the monoid element $M$, then the functor $f^{!}$is induced by an obvious functor $f_{M}: \mathcal{P} \mathcal{G}_{1}(E, p) \rightarrow \mathcal{P} \mathcal{G}_{1}\left(E^{\prime}, p^{\prime}\right)$ given on objects by $(K, e) \mapsto(M(K), f(e))$ and on morphisms by $(i, \omega) \mapsto\left(M(i), f_{*}(\omega)\right)$. This construction, of course, does depend on the choice of $M$, but for any two choices, say $M$ and $N$, the resulting functors can be compared by chosing an $L \geq M, N$ and comparing both of $f_{M}$ and $f_{N}$ to $f_{L}$ in a rather obvious way.

When comparing this with classical algebraic topology for pointed spaces, the $\mathcal{A}=\mathcal{A B}$ case of the above functor $f^{!}$is analogous to the functor between module categories

$$
f^{!}: \mathbb{Z}\left[\pi_{1}\left(E^{\prime}, e_{0}^{\prime}\right)\right]-\bmod \longrightarrow \mathbb{Z}\left[\pi_{1}\left(E, e_{0}\right)\right]-\bmod ,
$$

induced by a pointed map $f:\left(E, e_{0}\right) \rightarrow\left(E^{\prime}, e_{0}^{\prime}\right)$.

(Co)chains and (co)homology for a monoidally controlled space $(E, p)$, respectively for its universal cover $(E, p)^{\sim}$, are functors (to be defined later) that take values in the abelian category $\mathcal{A B}^{\mathcal{B}}\left(\Sigma_{\mathcal{M}}^{-1}\right)$, where $\mathcal{B}=\mathcal{P}$, respectively $\mathcal{B}=\mathcal{P} \mathcal{G}_{1}(E, p)$. In order to work efficiently in those categories we need to know when an object is (isomorphic to) zero and when a morphism is a mono- or epimorphism. Let $f: F \rightarrow G$ be a morphism in $\mathcal{A B}^{\mathcal{B}}\left(\Sigma_{\mathcal{M}}^{-1}\right)$. Thus $F, G: \mathcal{B} \rightarrow \mathcal{A B}$ are functors, and $f$ is represented, in the sense of Proposition 3.1, by a natural transformation $f_{M}: F \rightarrow G \circ M$ for some $M \in \mathcal{M}$. Recall that we write $\tau_{F}^{N}$ for the natural transformation $F(\alpha(u \leq N,-)): F \rightarrow F \circ N$ where $\alpha$ denotes the action of $\mathcal{M}$ on $\mathcal{B}$.

Proposition 3.3 ([5], Lemma 12.4 and 12.6)

(i) The object $F$ is a zero object if and only if there exists an $N \in \mathcal{M}$ such that $\tau_{F}^{N}$ is identically zero.

(ii) The morphism $f$ is a monomorphism if and only if it has a representative $f_{M}$ such that,

$$
\exists N \in \mathcal{M} \forall B \in \mathcal{B}: \operatorname{ker}\left[f_{M}: F(B) \rightarrow G(M B)\right] \subseteq \operatorname{ker}\left[\tau_{F}^{N}: F(B) \rightarrow F(N B)\right]
$$

(iii) The morphism $f$ is an epimorphism if and only if it has a representative $f_{M}$ such that,

$\exists N \in \mathcal{M} \forall B \in \mathcal{B}: \operatorname{im}\left[\tau_{G \circ M}^{N}: G(M B) \rightarrow G(M N B)\right] \subseteq \operatorname{im}\left[f_{M}: F(N B) \rightarrow G(M N B)\right]$

Remark 3.4 In [5], the natural transformation $f_{M}$ is called an eventual monomorphism, respectively an eventual epimorphism, if it has the property mentioned in (ii), respectively in (iii). 


\section{A Reduction to Cyclic Monoids}

In this section, we consider a control space $(Z, P, \mathcal{M})$ where $Z$ is assumed to be a connected, proper metric space, and we show how the categories related to this situation can be presented as colimits of similar categories where the monoids in question are cyclic. In [5], it is shown how the monoidally controlled world for any cyclic monoid coincides with the boundedly controlled world of [1] defined by a generator of the monoid. However, in the present exposition we shall stay with the monoidal terminology. Thus the heading of the present section might as well be: Cyclic Monoids Suffice - as long as your constructions are natural w.r.t. morphisms $M \subseteq N$ from $\mathcal{M}$.

An element $M \in \mathcal{M}$ is called symmetric if $(x, y) \in M \Leftrightarrow(y, x) \in M$. For any $N \in \mathcal{M}$, the composition $N N^{-1}$ is symmetric, and the collection $\left\{N N^{-1} \mid N \in \mathcal{M}\right\}$ is cofinal in the poset $\mathcal{M}$. For any $M \in \mathcal{M}-\{u\}$, let $\langle M\rangle$ denote the cyclic submonoid (with unit) of $\mathcal{M}$, generated by $M$. In Theorem 9.1 of [5], it is shown that for any symmetric $M \in \mathcal{M}-\{u\}$ the induced action of $\langle M\rangle$ on $P$ defines a control space $(Z, P,\langle M\rangle)$. Clearly, if $M \leq N$ (both symmetric), then we have inclusions of categories

$$
\mathcal{T O} \mathcal{P}^{\langle M\rangle} / Z \subseteq \mathcal{T} \mathcal{O} \mathcal{P}^{\langle N\rangle} / Z \subseteq \mathcal{T} \mathcal{O} \mathcal{P}^{\mathcal{M}} / Z
$$

where the objects are the same in all three cases. Moreover,

Lemma 4.1 ([5], Proposition 9.3) For $(Z, P, \mathcal{M})$ as above, the inclusions just mentioned give an isomorphism of categories

$$
\underset{M \in \mathcal{M}}{\lim _{\vec{T}}} \mathcal{T} \mathcal{O} \mathcal{P}^{\left\langle M M^{-1}\right\rangle} / Z \rightarrow \mathcal{T} \mathcal{O} \mathcal{P}^{\mathcal{M}} / Z
$$

which is the identity on objects.

Remark 4.2 Actually, in [5], the notation is slightly different, in that the monoidal control space $(Z, P,\langle M\rangle)$ is described as a bounded control space $(Z, P, M)$ in the sense of [1]. Also, the brackets in $\mathcal{T} \mathcal{O} \mathcal{P}^{\left\langle M M^{-1}\right\rangle} / Z$ are not included in [5]. Similar changes in notation apply in the following presentation of categories of the form $\mathcal{C}\left(\Sigma_{\mathcal{M}}^{-1}\right)$.

Clearly, if the monoid $\mathcal{M}$ acts on some category, $\mathcal{C}$, then for each $M \in \mathcal{M}$, the cyclic submonoid $\langle M\rangle$ also acts on $\mathcal{C}$ and one has the universal functor $Q_{\langle M\rangle}: \mathcal{C} \rightarrow \mathcal{C}\left(\Sigma_{\langle M\rangle}^{-1}\right)$ to the corresponding category of fractions. In section 5 of [5], it is shown that when $M \leq N$ there is an induced functor $\lambda_{M, N}: \mathcal{C}\left(\Sigma_{\langle M\rangle}^{-1}\right) \rightarrow \mathcal{C}\left(\Sigma_{\langle N\rangle}^{-1}\right)$ with $\lambda_{M, N} Q_{\langle M\rangle}=Q_{\langle N\rangle}$. Moreover, these functors define a direct system of categories and one has

Lemma 4.3 ([5], Theorem 5.2) There is an isomorphism of categories

$$
\lambda: \lim _{M \in \mathcal{M}} \mathcal{C}\left(\Sigma_{\langle M\rangle}^{-1}\right) \rightarrow \mathcal{C}\left(\Sigma_{\mathcal{M}}^{-1}\right)
$$

Remark 4.4 We note that the functors $\lambda_{M, N}$ and $\lambda$ are the identity on objects, and when morphisms are given by representatives as in Proposition 3.1, the functor $\lambda$ preserves representatives. Also note that one may replace $\mathcal{C}\left(\Sigma_{\langle M\rangle}^{-1}\right)$ by $\mathcal{C}\left(\Sigma_{\left\langle M M^{-1}\right\rangle}^{-1}\right)$ to facilitate the simultaneous use of Lemma 4.1 and Lemma 4.3. 


\section{The Universal Cover of a Controlled Space}

Let $(Z, P, \mathcal{M})$ be a control space and let $(E, p)$ be a space controlled over $Z$. The universal cover of $(E, p)$ will be denoted $(E, p)^{\sim}$. It is not a space controlled over $Z$ but rather (in the terminology of [5] and [1]) a fragmented space over $\mathcal{P} \mathcal{G}_{1}(E, p)$, i.e., $(E, p)^{\sim}$ is an object in the category $\mathcal{T} \mathcal{O P}^{\mathcal{P} \mathcal{G}_{1}(E, p)}\left(\Sigma_{\mathcal{M}}^{-1}\right)$. To recall the definition, for any pointed topological space $\left(W, w_{0}\right)$, we let $\left(W, w_{0}\right)^{\sim}=\left(W, w_{0}\right)^{(I, 0)} / \sim$ be the space of homotopy classes, rel endpoints, of paths in $W$ starting at $w_{0}$. Clearly, this construction is functorial w.r.t. continuous based maps, and if $W$ is semi-locally simply connected and locally path connected, then $\left(W, w_{0}\right)^{\sim}$ is the standard construction of the universal covering of the path component of $W$ containing $w_{0}$, see e.g. [7].

Definition 5.1 The universal cover $(E, p)^{\sim} \in\left|\mathcal{T} \mathcal{O P}^{\mathcal{P} \mathcal{G}_{1}(E, p)}\left(\Sigma_{\mathcal{M}}^{-1}\right)\right|$, is defined by

(i) $(E, p)^{\sim}(K, e)=\left(p^{-1} K, e\right)^{\sim}, \quad(K, e) \in\left|\mathcal{P} \mathcal{G}_{1}(E, p)\right|$,

(ii) $(E, p)^{\sim}(i, \omega)=\omega_{\#} \circ i_{*}:\left(p^{-1} K, e\right)^{\sim} \rightarrow\left(p^{-1} K^{\prime}, e^{\prime}\right)^{\sim}, \quad(i, \omega):(K, e) \rightarrow\left(K^{\prime}, e^{\prime}\right)$.

Here, $i: p^{-1} K \rightarrow p^{-1} K^{\prime}$ is the inclusion, and $\omega_{\#}$ is given by pre-concatenation with the pathclass $\omega$.

We insist on considering $(E, p)^{\sim}$ an object in the category of fractions $\mathcal{T} \mathcal{O P}^{\mathcal{P} \mathcal{G}_{1}(E, p)}\left(\Sigma_{\mathcal{M}}^{-1}\right)$ rather than the functor category $\mathcal{T} \mathcal{O P}^{\mathcal{P G}} \mathcal{G}_{1}(E, p)$ itself because that makes the construction $(E, p) \mapsto(E, p)^{\sim}$ "functorial" in the following weak sense. If $f:(E, p) \rightarrow\left(E^{\prime}, p^{\prime}\right)$ is controlled by $M \in \mathcal{M}$, then we have the functor $f^{!}: \mathcal{T} \mathcal{O P}^{\mathcal{P} \mathcal{G}_{1}\left(E^{\prime}, p^{\prime}\right)}\left(\Sigma_{\mathcal{M}}^{-1}\right) \rightarrow \mathcal{T} \mathcal{O P}^{\mathcal{P G}_{1}(E, p)}\left(\Sigma_{\mathcal{M}}^{-1}\right)$, described in Proposition 3.2, and $f$ induces a morphism $f_{*}:(E, p)^{\sim} \rightarrow f^{!}\left(\left(E^{\prime}, p^{\prime}\right)^{\sim}\right)$. We recall that $f^{!}$is only defined up to a canonical natural equivalence, and we leave it to the interested reader to figure out precisely how functorial the morphism $f_{*}$ is, mentioning only that $f_{*}$ is represented (in the sense of Proposition 3.1) by a natural transformation $(E, p)^{\sim} \rightarrow f^{!}\left(\left(E^{\prime}, p^{\prime}\right)^{\sim}\right)$ having

$$
f_{(K, e)}=\left(f \mid p^{-1} K\right)_{*}:\left(p^{-1} K, e\right)^{\sim} \rightarrow\left(p^{\prime-1} M K^{\prime}, f(e)\right)^{\sim}, \quad(K, e) \in\left|\mathcal{P} \mathcal{G}_{1}(E, p)\right| .
$$

If $(E, p)$ is a controlled CW complex over $Z$, then for each $K \in \mathcal{P}$ there is a smallest subcomplex of $E$ containing $p^{-1} K$. We call it $c w\left(p^{-1} K\right)$, and we note that when $K \subseteq K^{\prime}$ one has $c w\left(p^{-1} K\right) \subseteq c w\left(p^{-1} K^{\prime}\right)$. Moreover, since the cells of $E$ are bounded by some $M \in \mathcal{M}$ and since $E$ is finite dimensional, there is an $L \in \mathcal{M}$ such that

$$
p^{-1} K \subseteq c w\left(p^{-1} K\right) \subseteq p^{-1} L K
$$

for each $K \in \mathcal{P}$. Also, if $f:(E, p) \rightarrow\left(E^{\prime}, p^{\prime}\right)$ is a controlled, cellular map between controlled CW complexes, then for some $L \in \mathcal{M}$ (depending on the bound of $f$, and the dimension of $E)$, one has $f\left(c w\left(p^{-1} K\right)\right) \subseteq c w\left(p^{-1} L K\right)$. Now, in the construction of $(E, p)^{\sim}$, one may replace each $p^{-1} K$ by $c w\left(p^{-1} K\right)$. There results a variation on the universal cover construction to be denoted $(E, p)^{\widetilde{c w}}$. The inclusions displayed in the above are then easily seen to imply that $(E, p)^{\sim}$ and $(E, p)^{\widetilde{c w}}$ are canonically isomorphic in the category $\mathcal{T} \mathcal{O P}^{\mathcal{P} \mathcal{G}_{1}(E, p)}\left(\Sigma_{\mathcal{M}}^{-1}\right)$. 
Remark 5.2 In [5] the functors $K \mapsto p^{-1} K$ and $K \mapsto c w\left(p^{1} K\right)$ are treated in section 10 under the names "the inverse image fragmentation of $(E, p)$ ", respectively, "the smallest subcomplex fragmentation of $(E, p) . "$

\section{Controlled Chains and Homology}

Let $(Z, P, \mathcal{M})$ be a control space, and let $(E, p)$ be a space controlled over $Z$ or a $\mathrm{CW}$ complex controlled over $Z$. In this section we recall the definition of the (singular or cellular, as the case may be) chain complex and the homology of $(E, p)$ and of its universal cover. We shall use $\Delta$ and $C$ to denote classical singular and cellular chains, respectively. For lack of a better terminology, we shall talk of chain "groups" and homology "groups" even though they are not groups at all.

Definition 6.1 The singular chain "group" $\Delta_{n}^{\mathcal{M}}$, and the cellular chain "group" $C_{n}^{\mathcal{M}}$, are functors

$$
\Delta_{n}^{\mathcal{M}}: \mathcal{T} \mathcal{O} \mathcal{P}^{\mathcal{M}} / Z \rightarrow \mathcal{A B}^{\mathcal{P}}\left(\Sigma_{\mathcal{M}}^{-1}\right), \text { and } C_{n}^{\mathcal{M}}: \mathcal{C W}^{\mathcal{M}} / Z \rightarrow \mathcal{A} \mathcal{B}^{\mathcal{P}}\left(\Sigma_{\mathcal{M}}^{-1}\right)
$$

They are defined as follows: For an arbitrary object $K$ of $\mathcal{P}$ one has

$$
\Delta_{n}^{\mathcal{M}}(E, p): K \mapsto \Delta_{n}\left(p^{-1} K\right) \text {, and } C_{n}^{\mathcal{M}}(E, p): K \mapsto C_{n}\left(c w\left(p^{-1} K\right) .\right.
$$

The boundary morphisms are represented by the obvious natural transformations

$$
\partial_{n}: \Delta_{n}\left(p^{-1}-\right) \rightarrow \Delta_{n-1}\left(p^{-1}-\right), \text { and } \partial_{n}: C_{n}\left(c w\left(p^{-1}-\right)\right) \rightarrow C_{n-1}\left(c w\left(p^{-1}-\right)\right) .
$$

Finally, if $f:(E, p) \rightarrow\left(E^{\prime}, p^{\prime}\right)$ is a continuous map controlled by a monoid element $M \in \mathcal{M}$, then the morphism $f_{*}: \Delta_{n}^{\mathcal{M}}(E, p) \rightarrow \Delta_{n}^{\mathcal{M}}\left(E^{\prime}, p^{\prime}\right)$ is represented by the natural transformation

$$
\Delta\left(f \mid p^{-1}-\right): \Delta_{n}\left(p^{-1}-\right) \rightarrow \Delta_{n}\left(p^{-1} M-\right) .
$$

A similar definition holds for $f_{*}: C_{n}^{\mathcal{M}}(E, p) \rightarrow C_{n}^{\mathcal{M}}\left(E^{\prime}, p^{\prime}\right)$, if we are dealing with a continuous, cellular map $f:(E, p) \rightarrow\left(E^{\prime}, p^{\prime}\right)$, controlled by $M$.

Remark 6.2 Both of these chain functors take values in an abelian category, so one may pass to the (singular or cellular) homology "groups". As usual, when both of them make sense, they are canonically isomorphic (as objects in $\mathcal{A B}^{\mathcal{P}}\left(\Sigma_{\mathcal{M}}^{-1}\right)$ ), so we shall introduce only one notation, namely $H_{n}^{\mathcal{M}}(E, p)$. Depending on the context, one has

$$
H_{n}^{\mathcal{M}}(E, p)=H_{n}\left(p^{-1}-\right): \mathcal{P} \rightarrow \mathcal{A B}, \text { or } H_{n}^{\mathcal{M}}(E, p)=H_{n}\left(c w\left(p^{-1}-\right)\right): \mathcal{P} \rightarrow \mathcal{A B} .
$$

We next pass to the universal cover $(E, p)^{\sim}$ or $(E, p)^{\widetilde{c w}}$ as the case may be. We shall stick to the former case and the singular chains. The reader should easily adapt the treatment to the $\mathrm{CW}$ case. 
Definition 6.3 The n'th singular chain "group" of the universal cover of $(E, p)$ is denoted $\Delta_{n}^{\mathcal{M}}\left((E, p)^{\sim}\right)$. It is an object in the abelian category $\mathcal{A B}^{\mathcal{P} \mathcal{G}_{1}(E, p)}\left(\Sigma_{\mathcal{M}}^{-1}\right)$, and it is defined to be simply the composite functor

$$
\Delta_{n} \circ(E, p)^{\sim}: \mathcal{P} \mathcal{G}_{1}(E, p) \rightarrow \mathcal{A B}
$$

The boundary morphism is represented by the natural transformation $\partial_{n} \circ(E, p)^{\sim}$.

Remark 6.4 This chain "group" is functorial only in the same weak sense as the universal cover, i.e., a controlled morphism $f:(E, p) \rightarrow\left(E^{\prime}, p^{\prime}\right)$ gives rise to an exact functor

$$
f^{!}: \mathcal{A B}^{\mathcal{P} \mathcal{G}_{1}\left(E^{\prime}, p^{\prime}\right)}\left(\Sigma_{\mathcal{M}}^{-1}\right) \rightarrow \mathcal{A B}^{\mathcal{P} \mathcal{G}_{1}(E, p)}\left(\Sigma_{\mathcal{M}}^{-1}\right)
$$

and a morphism $f_{*}: \Delta_{n}^{\mathcal{M}}(E, p) \rightarrow f^{!}\left(\Delta_{n}^{\mathcal{M}}\left(E^{\prime}, p^{\prime}\right)\right)$.

Of course, the homology "group", $H_{n}^{\mathcal{M}}\left((E, p)^{\sim}\right)$ of this chain complex is functorial in the same sense. It may be concretely interpreted as the composite functor

$$
H_{n}^{\mathcal{M}}\left((E, p)^{\sim}\right)=H_{n} \circ(E, p)^{\sim}: \mathcal{P} \mathcal{G}_{1}(E, p) \rightarrow \mathcal{A B} .
$$

Remark 6.5 In [5], the cellular chains and the homology of the universal cover $(E, p)^{\widetilde{c w}}$ are defined to live in a variation of the category $\mathcal{A B}^{\mathcal{P} \mathcal{G}_{1}(E, p)}$. The variation is of no consequence since the two categories are canonically equivalent. For completeness, though, we note that the difference arises when one defines the fundamental groupoid functor $\mathcal{G}_{1}(E, p): \mathcal{P} \rightarrow$ $\mathcal{G P O I D}$. In the present paper, this always takes $K$ to the fundamental groupoid of $p^{-1} K$. In [5], we use the fundamental groupoid of the smallest CW complex, $c w\left(p^{-1} K\right)$ instead, when we deal with a controlled CW complex.

\section{$7 \quad N$-small chains and locally finite chains}

Our controlled cochains will not be functionals defined on all chains, but rather on the so called $N$-small chains only. Here $N$ can be any monoid element $N \in \mathcal{M}-\{u\}$.

Proposition 7.1 Let $(E, p) \in\left|\mathcal{T} \mathcal{O P}^{\mathcal{M}} / Z\right|$. Let $N \in \mathcal{M}-\{u\}$ and put $L=N N^{-1}$. There is a subcomplex $\Delta_{*}^{N}(E) \subseteq \Delta_{*}(E)$ such that

(i) For any $X \subseteq E$, the inclusion $\Delta_{*}^{N}(X):=\Delta_{*}^{N}(E) \cap \Delta_{*}(X) \subseteq \Delta_{*}(X)$ is a chain equivalence.

(ii) For any singular simplex $\sigma \in \Delta_{q}^{N}(E)$ and any $K \in P$, if $\sigma\left(\Delta^{q}\right) \cap p^{-1} K \neq \emptyset$, then $\sigma\left(\Delta^{q}\right) \subseteq p^{-1} L K$

Proof : We let $\mathcal{V}_{N}=\left\{p^{-1} N(p(e)) \mid e \in E\right\}$. Since $N$ is a reflexive relation and an open subset of $Z \times Z$ and since $p$ is continuous, $E \subseteq \bigcup_{U \in \mathcal{V}_{N}} \operatorname{int}(U)$. Now by any basic textbook on algebraic topology, like [12, Thm. 1.14], $\Delta_{*}(E)$ is chain equivalent to $\Delta_{*}\left(\mathcal{V}_{N}\right)$, where the latter is the subcomplex generated by the set of singular simplices $\sigma: \Delta^{q} \rightarrow E$ 
for which there exists $U \in \mathcal{V}_{N}$ such that $\sigma\left(\Delta^{q}\right) \subseteq U$. Moreover, the same reference shows that $\Delta_{*}\left(\mathcal{V}_{N}\right) \cap \Delta_{*}(X) \subseteq \Delta_{*}(X)$ is a chain equivalence for any $X \subseteq E$. Finally, we claim that $\Delta_{q}\left(\mathcal{V}_{N}\right)$ satisfies (ii) for $L=N N^{-1}$. Indeed, if $\sigma$ is a generator for $\Delta_{q}\left(\mathcal{V}_{N}\right)$, then by definition there exists $e \in E$ such that $\sigma\left(\Delta^{q}\right) \subseteq p^{-1} N(p(e))$. Assume that for some $K \in P$ we have $\sigma\left(\Delta^{q}\right) \cap p^{-1} K \neq \emptyset$, and let $x \in \sigma\left(\Delta^{q}\right) \cap p^{-1} K$. Then $p(x) \in N(p(e)) \cap K$, so $p(e) \in N^{-1}(K)$ whereby $\sigma\left(\Delta^{q}\right) \subseteq p^{-1} N N^{-1}(K)$.

We call the elements of $\Delta_{*}^{N}(E) N$-small singular chains on $E$. Since property (i) holds for subsets of the form $p^{-1} K, K \in P$, we may restrict to such $N$-small chains when we define the $\mathcal{M}$-controlled singular chains as in section 6 . This leads to a subcomplex in the category $\mathcal{A B}^{\mathcal{P}}\left(\Sigma_{\mathcal{M}}^{-1}\right)$

$$
\Delta_{*}^{\mathcal{M}, N}(E, p) \subseteq \Delta_{*}^{\mathcal{M}}(E, p) .
$$

Similarly, when we define the singular chains of the universal cover, $\Delta_{*}^{\mathcal{M}}\left((E, p)^{\sim}\right)$, for each $(K, e) \in\left|\mathcal{P G}_{1}(E, p)\right|$, we may restrict to those singular simplices in $\left(p^{-1} K, e\right)^{\sim}$ which are lifts of $N$-small simplices in $p^{-1} K$. There results a subcomplex in $\mathcal{A B}^{\mathcal{P} \mathcal{G}_{1}(E, p)}\left(\Sigma_{\mathcal{M}}^{-1}\right)$

$$
\Delta_{*}^{\mathcal{M}, N}\left((E, p)^{\sim}\right) \subseteq \Delta_{*}^{\mathcal{M}}\left((E, p)^{\Upsilon}\right) .
$$

Clearly the above inclusions are homology isomorphims already at the functor category level and hence, a fortiori, in the categories of fractions $\mathcal{A B}^{\mathcal{P}}\left(\Sigma_{\mathcal{M}}^{-1}\right)$ ), respectively $\left.\mathcal{A B}^{\mathcal{P} \mathcal{G}_{1}(E, p)}\left(\Sigma_{\mathcal{M}}^{-1}\right)\right)$. Actually, more is true.

Proposition 7.2 The chain complexes $\Delta_{*}^{\mathcal{M}, N}(E, p)$ and $\Delta_{*}^{\mathcal{M}, N}\left((E, p)^{\sim}\right)$ are independent of $N$ up to chain homotopy equivalence.

Proof : We outline the proof in the case of the universal cover. The other case is similar. It suffices to show that the inclusion-induced morphism $i: \Delta_{*}^{\mathcal{M}, N}\left((E, p)^{\sim}\right) \subseteq \Delta_{*}^{\mathcal{M}, \bar{N}}\left((E, p)^{\sim}\right)$ is a chain homotopy equivalence whenever $N \subseteq \bar{N}$. Now, in any abelian category, a chain map between projective chain complexes is a chain homotopy equivalence if and only if it is a homology isomorphism. For the case of free chain complexes of modules over a ring, this is Theorem 9.8 of [10], and the proof given there generalizes immediately to our situation. Since $i$ is a homology isomorphism, it will be sufficient to show that each chain "group" $\Delta_{*}^{N}$ is projective in $\mathcal{A B}^{\mathcal{P} \mathcal{G}_{1}(E, p)}\left(\Sigma_{\mathcal{M}}^{-1}\right)$. The proof of this is outlined in the appendix.

We also need to consider the chain complex $\Delta_{*}^{l f, N}(E)$ of locally finite, $N$-small chains on $E$. Such a chain, in dimension $k$, say, is a formal (integral) linear combination of all $N$-small singular $k$-simplices in $E, \sum_{\sigma} n_{\sigma} \sigma$, with the property that each compact subset meets at most finitely many $\sigma\left(\Delta^{k}\right)$ with $n_{\sigma} \neq 0$. We shall consider two types of transfer maps connecting such groups, both of them under the assumption that $E$ is locally path connected and semilocally one-connected.

First, for $B \subseteq E$ an open subspace we denote by $q_{B}: \widetilde{B} \rightarrow E$ the universal covering map for $B$ followed by the inclusion into $E$. The corresponding transfer map

$$
q_{B}^{\#}: \Delta_{k}^{l f, N}(E) \rightarrow \Delta_{k}^{l f, N}(\widetilde{B})
$$


is defined by requiring that it preserve formal linear combinations and that it map any generator $\sigma$ to the formal sum of all its liftings $\tilde{\sigma}$ through the map $q_{B}$. The covering property of $q_{B}$ is used to ensure local finiteness of each image under $q_{B}^{\#}$. In applications, $\widetilde{B}$ will be of the form $\left(p^{-1} L K, e\right)^{\sim}$, i.e., $B$ is the component of $p^{-1} L K$ containing the point $e$. Here $L$ is always understood to be $N N^{-1}$. For such instances, the subscript $B$ will often have to be inferred from the context.

Next, consider a morphism $(i, \omega):(K, e) \rightarrow\left(K^{\prime}, e^{\prime}\right)$ in the category $\mathcal{P} \mathcal{G}_{1}(E, p)$. It induces a map $(L(i), \omega)^{\sim}:\left(p^{-1} L K, e\right)^{\sim} \rightarrow\left(p^{-1} L K^{\prime}, e^{\prime}\right)^{\sim}$. And this in turn gives rise to the transfer map, defined as above

$$
\left.(L(i), \omega)^{\#}: \Delta_{*}^{l f, N}\left(p^{-1} L K^{\prime}, e^{\prime}\right)^{\sim}\right) \rightarrow \Delta_{*}^{l f, N}\left(\left(p^{-1} L K, e\right)^{\sim}\right) .
$$

It is now easy to prove the following result.

Proposition 7.3 If $E$ is locally path connected and semilocally one-connected, then the maps $q_{B}^{\#}$ and $(L(i), \omega)^{\#}$ are well defined, but (of course) in general they are not chain maps. Moreover, for any pair of morphisms in $\mathcal{P G}_{1}(E, p)$

$$
(K, e) \underset{(i, \omega)}{\longrightarrow}\left(K^{\prime}, e^{\prime}\right) \underset{\left(i^{\prime}, \omega^{\prime}\right)}{\longrightarrow}\left(K^{\prime \prime}, e^{\prime \prime}\right)
$$

the following diagram commutes.

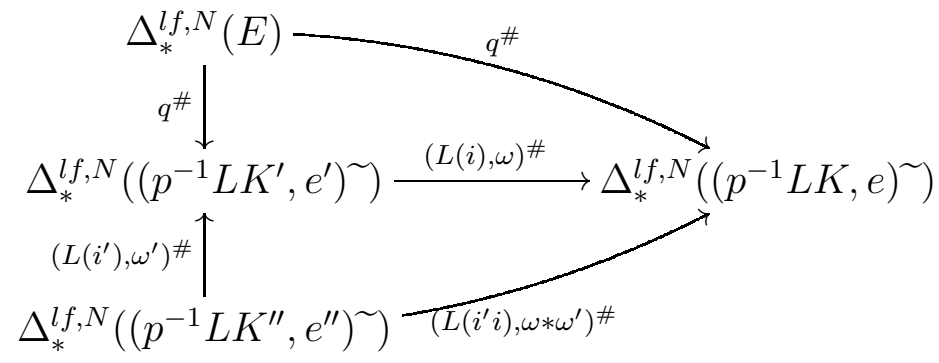

Remark 7.4 The reader should have no trouble adapting the above discussion to the cellular case. Indeed, the cells of any $(E, p)$ are assumed to be bounded by some fixed $N$ so we do not need to artificially restrict the size of the generators of our chains.

\section{Controlled cochains and cohomology, "upstairs"}

We use the word upstairs to indicate that we treat cochains and cohomology at the universal cover level. In order for this to work out well, we assume that $(E, p)$ has $E$ locally path connected and semi locally simply connected. We shall treat only the case ofsingular cochians. Thus we assume given a monoid element $N$ and we let $L=N N^{-1}$ as in the preceding section.

Recall that for any $(K, e) \in \mathcal{P G}_{1}(E, p),\left(p^{-1} L K, e\right)^{\sim}$ is the universal cover of the component of $p^{-1} L K$ which contains $e$. There is an induced covering $\left(p^{-1} K, e\right)^{-}$over the $e$ 
component of the subspace $p^{-1} K \subseteq p^{-1} L K$. For brevity, we write $\complement\left(p^{-1} K, e\right)^{-}$for the complement of $\left(p^{-1} K, e\right)^{-}$in $\left(p^{-1} L K, e\right)^{\sim}$, and we consider the relative cochain complex $\Delta_{N}^{*}\left(\left(p^{-1} L K, e\right)^{\sim}, \complement \complement\left(p^{-1} K, e\right)^{-}\right)$, where the subscript $N$ indicates that the domain is the $N$-small chain complex $\Delta_{*}^{N}\left(\left(p^{-1} L K, e\right)^{\sim}\right)$.

Definition 8.1 A cochain $\varphi \in \Delta_{N}^{q}\left(\left(p^{-1} L K, e\right)^{\sim}, \complement\left(p^{-1} K, e\right)^{-}\right)$is said to be compactly supported, see e.g. [9], if there exists a compact subset $C \subseteq\left(p^{-1} K, e\right)^{-}$such that the following implication holds for any $N$-small singular simplex $\sigma: \Delta^{q} \rightarrow\left(p^{-1} L K, e\right)^{\sim}$,

$$
\varphi(\sigma) \neq 0 \Rightarrow \sigma\left(\Delta^{q}\right) \cap C \neq \emptyset
$$

i.e., $\varphi$ belongs to the subgroup $\Delta_{N}^{q}\left(\left(p^{-1} L K, e\right)^{\sim}, \complement C\right)$ for some compact $C \subseteq\left(p^{-1} K, e\right)^{-}$. We write $\Delta_{c, N}^{q}\left(\left(p^{-1} L K, e\right)^{\sim}, \complement\left(p^{-1} K, e\right)^{-}\right)$for the set of such cochains.

The following Proposition is now easy to prove.

Proposition 8.2 Compactly supported cochains form a subcomplex

$$
\Delta_{c, N}^{*}\left(\left(p^{-1} L K, e\right)^{\sim}, \complement\left(p^{-1} K, e\right)^{-}\right) \subseteq \Delta_{N}^{q}\left(\left(p^{-1} L K, e\right)^{\sim}, \complement\left(p^{-1} K, e\right)^{-}\right) .
$$

Any cochain $\varphi: \Delta_{q}^{N}\left(\left(p^{-1} L K, e\right)^{\sim}\right) \rightarrow \mathbb{Z}$ in this subcomplex extends uniquely to a cochain $\varphi^{l f}: \Delta_{q}^{l f, N}\left(\left(p^{-1} L K, e\right)^{\sim}\right) \rightarrow \mathbb{Z}$. Therefore, the transfer map $(L(i), \omega)^{\#}$ of Proposition 7.3 can be dualized to a map

$$
(L(i), \omega)_{\#}: \Delta_{N}^{*}\left(\left(p^{-1} L K, e\right)^{\sim}, \complement\left(p^{-1} K, e\right)^{-}\right) \longrightarrow \Delta_{N}^{*}\left(\left(p^{-1} L K^{\prime}, e^{\prime}\right)^{\sim}, \complement\left(p^{-1} K^{\prime}, e^{\prime}\right)^{-}\right) .
$$

This map commutes with the coboundary maps, so the assigmnent

$$
\Delta_{c, N}^{*}\left((E, p)^{\sim}\right):(K, e) \mapsto \Delta_{c, N}^{*}\left(\left(p^{-1} L K, e\right)^{\sim}, \complement\left(p^{-1} K, e\right)^{-}\right)
$$

defines a cochain complex $\Delta_{c, N}^{*}\left((E, p)^{\sim}\right)$ in the functor category $\mathcal{A B}^{\mathcal{P} \mathcal{G}_{1}(E, p)}$.

Proof : The proof is purely formal except for the fact that the transfer map on locally finite chains, $(L(i), \omega)^{\#}$, does not commute with differentials, so we have to see why the diagram

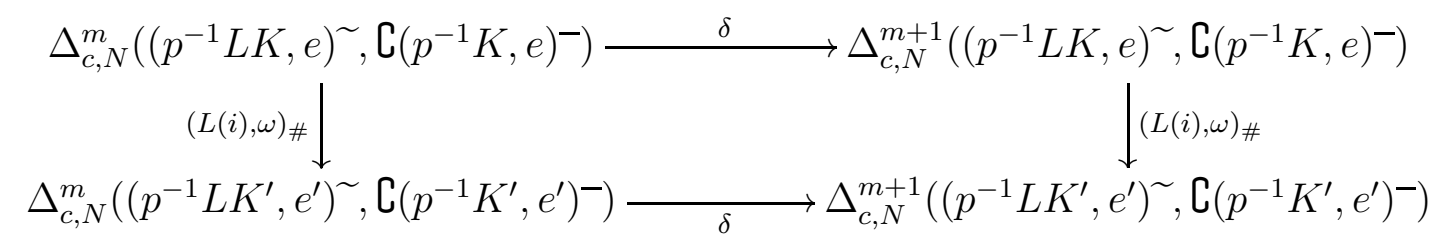

commutes anyway. We start with an element $\varphi$ in the upper left hand corner.

For an arbitrary $N$-small, singular simplex $\rho^{\prime}: \Delta^{m+1} \rightarrow\left(p^{-1} L K^{\prime}, e^{\prime}\right)^{\sim}$, a direct computation gives the formulae

$$
\delta(L(i), \omega)_{\#}(\varphi)\left(\rho^{\prime}\right)=\sum_{(j, \sigma) \in A}(-1)^{j} \varphi(\sigma), \quad(L(i), \omega)_{\#}(\delta \varphi)\left(\rho^{\prime}\right)=\sum_{(k, \rho) \in B}(-1)^{k} \varphi\left(\rho^{(k)}\right),
$$


where the index sets are defined as follows (as usual, ${ }^{(j)}$ indicates the face opposite of vertex number $j$ )

$$
\begin{aligned}
& A=\left\{(j, \sigma) \mid j=0,1, \cdots, m+1 ; \sigma \in \Delta_{m}^{N}\left(\left(p^{-1} L K, e\right)^{\sim}\right) ;(i, \omega)_{\#}(\sigma)=\rho^{\prime(j)}\right\}, \\
& B=\left\{(k, \rho) \mid k=0,1, \cdots, m+1 ; \rho \in \Delta_{m+1}^{N}\left(\left(p^{-1} L K, e\right)^{\sim}\right) ;(i, \omega)_{*}(\rho)=\rho^{\prime}\right\} .
\end{aligned}
$$

The map $\alpha: B \rightarrow A$ with $\alpha(k, \rho)=\left(k, \rho^{(k)}\right)$ is easily seen to be injective, and the summands corresponding to the indices $(k, \rho)$ and $\alpha(k, \rho)$ are equal, so we only need to show that

$$
\alpha(B) \supseteq\{(j, \sigma) \in A \mid \varphi(\sigma) \neq 0\} .
$$

Therefore, let $(j, \sigma) \in A$. Then the fully drawn arrows in the following diagram commute ( $q$ and $q^{\prime}$ denote universal covering maps followed by inclusions), and we have to fill in the dotted arrow preserving commutativity under the asumption that $\varphi(\sigma) \neq 0$.

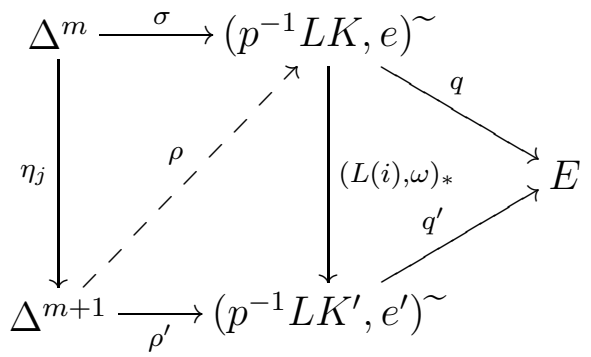

Since $\varphi$ is a relative cochain, $\varphi(\sigma)$ being non zero implies that $q \sigma\left(\Delta^{m}\right) \cap p^{-1} K \neq \emptyset$. But then also $q^{\prime} \rho^{\prime}\left(\Delta^{m+1}\right) \cap p^{-1} K \neq \emptyset$ and since $\rho^{\prime}$ is $N$-small, it follows that $q^{\prime} \rho^{\prime}\left(\Delta^{m+1}\right) \subseteq p^{-1} L K$. Since $q$ is a covering map onto its image, and $\sigma$ lifts the restriction $q^{\prime} \rho^{\prime} \eta_{j}$, there is a unique $\rho$ with $\rho \eta_{j}=\sigma$ and $q \rho=q^{\prime} \rho^{\prime}$. And $(i, \omega)_{*} \rho=\rho^{\prime}$ now follows because these two maps agree on $\eta_{j}\left(\Delta^{m}\right)$ and meet under composition with $q^{\prime}$.

Definition 8.3 The controlled cochain complex of $(E, p)^{\sim}$ will be denoted $\Delta_{\mathcal{M}, N}^{*}\left((E, p)^{\sim}\right)$. It is defined to be the image of the complex $\Delta_{c, N}^{*}\left((E, p)^{\sim}\right)$ under the quotient functor $Q$ : $\mathcal{A B}^{\mathcal{P} \mathcal{G}_{1}(E, p)} \rightarrow \mathcal{A B}^{\mathcal{P} \mathcal{G}_{1}(E, p)}\left(\Sigma_{\mathcal{M}}^{-1}\right)$.

The homology of this complex is denoted $H_{\mathcal{M}, N}^{*}\left((E, p)^{\sim}\right)$ and is called the controlled cohomology of $(E, p)^{\sim}$.

Remark 8.4 Of course, the "group" $H_{\mathcal{M}, N}^{m}\left((E, p)^{\sim}\right)$ is simply the functor $\mathcal{P} \mathcal{G}_{1}(E, p) \rightarrow$ $\mathcal{A B}$ which takes $(K, e)$ to

$$
H_{c, N}^{m}\left(\left(p^{-1} L K, e\right)^{\sim}, \complement\left(p^{-1} K, e\right)^{-}\right):=\underset{C}{\lim _{\longrightarrow}} H_{N}^{q}\left(\left(p^{-1} L K, e\right)^{\sim}, \complement C\right)
$$

where $C$ ranges over all compact sets $C \subseteq\left(p^{-1} K, e\right)^{-}$. In particular, a different choice for $N$ will give rise to isomorphic cohomology "groups". 


\section{The controlled cap product}

As usual, let $(E, p)$ be a space controlled over $(Z, \mathcal{P}, \mathcal{M})$. As in the above a mononoid element $N \neq u$ has been chosen, and we have $L=N N^{-1}$

Proposition 9.1 Any $N$-small chain $z \in \Delta_{m}^{l f, N}(E)$ gives rise to a graded morphism

$$
-\cap z: \Delta_{\mathcal{M}, N}^{*}\left((E, p)^{\sim}\right) \longrightarrow \Delta_{m-*}^{\mathcal{M}, N}\left((E, p)^{\sim}\right)
$$

in the category $\mathcal{A B}^{\mathcal{P} \mathcal{G}_{1}(E, p)}\left(\Sigma_{\mathcal{M}}^{-1}\right)$. The following identity holds.

$$
\partial \circ(-\cap z)=(-\cap z) \circ \delta+(-1)^{q}(-\cap \partial z): \Delta_{\mathcal{M}, N}^{q}\left((E, p)^{\sim}\right) \longrightarrow \Delta_{m-q}^{\mathcal{M}, N}\left((E, p)^{\sim}\right) .
$$

Proof : The morphism $-\cap z$ will be represented by a natural transformation

$$
(-\cap z)_{L}: \Delta_{\mathcal{M}, N}^{*}\left((E, p)^{\sim}\right) \longrightarrow \Delta_{m-*}^{\mathcal{M}, N}\left((E, p)^{\sim}\right) \circ L
$$

the instance of which at $(K, e)$ is given as follows: The covering map $q_{(L K, e)}:\left(p^{-1} L K, e\right)^{\sim} \rightarrow$ $E$ induces a transfer map as in section 7 , so we have the locally finite chain

$$
q_{(L K, e)}^{\#}(z) \in \Delta_{m}^{l f, N}\left(\left(p^{-1} L K, e\right)^{\sim}\right) .
$$

The desired natural transformation is now the classical cap product

$$
-\cap q_{(L K, e)}^{\#}(z): \Delta_{c, N}^{*}\left(\left(p^{-1} L K, e\right)^{\sim}, \complement\left(p^{-1} K, e\right)^{-}\right) \longrightarrow \Delta_{m-*}^{L}\left(\left(p^{-1} L K, e\right)^{\sim}\right) .
$$

Naturality of this construction viz-a-viz morphisms induced by $(i, \omega):(K, e) \rightarrow\left(K^{\prime}, e^{\prime}\right)$ is an easy consequence of the commutative top triangle in Proposition 7.3 in combination with the well known classical cap product naturality of the form $f_{*}\left(f^{*}(a) \cap b\right)=a \cap f_{*}(b)$.

To prove the boundary-coboundary identity, let $\varphi \in \Delta_{c, N}^{q}\left(\left(p^{-1} L K, e\right)^{\sim}, \complement\left(p^{-1} K, e\right)^{-}\right)$be given. There is then the classical identity

$$
\partial\left(\varphi \cap q_{(L K, e)}^{\#}(z)\right)=\delta \varphi \cap q_{(L K, e)}^{\#}(z)+(-1)^{q} \varphi \cap \partial q_{(L K, e)}^{\#}(z),
$$

so we only have to prove that $\varphi \cap \partial q_{(L K, e)}^{\#}(z)=\varphi \cap q_{(L K, e)}^{\#}(\partial z)$. But $\varphi$ vanishes on chains in the complement $\complement\left(p^{-1} K, e\right)^{-} \subseteq\left(p^{-1} L K, e\right)^{\sim}$, so it suffices to show that the difference $\partial q_{(L K, e)}^{\#}(z)-q_{(L K, e)}^{\#}(\partial z)$ is supported in that complement Clearly it suffices to treat the case where $z=\sigma: \Delta^{q} \rightarrow E$ is any $N$-small singular simplex in $E$. Then a simple computation reveals the formula

$$
\partial q_{(L K, e)}^{\#}(\sigma)-q_{(L K, e)}^{\#} \partial(\sigma)=\sum_{i=0}^{q}(-1)^{i}\left[\sum_{\tau} \tau^{(i)}-\sum_{\rho_{i}} \rho_{i}\right] .
$$

Here, $\tau$ ranges over all lifts of $\sigma$ through $q_{(L K, e)}$ while each $\rho_{i}$ ranges over all lifts of the face $\sigma^{(i)}$ through the same map. Now, if $\tau$ lifts $\sigma$ then $\tau^{(i)}$ apppears as a $\rho_{i}$, so the only terms surviving in the expression are (up to the minus sign) those $\rho_{i}$, which lift a face $\sigma^{(i)}$ for which the whole simplex, $\sigma$ does not lift. But when a face of an $N$-small singular simplex lifts and the whole simplex does not, then the simplex in question cannot meet $p^{-1} K$. This completes the proof. 


\section{Controlled Poincaré Duality, "upstairs"}

Here, finally, is the main result of the present paper. In it, $(E, p)$ is a controlled space over a control structure $(Z, \mathcal{P}, \mathcal{M})$. Moreover $E$ is a connected $n$-manifold which is oriented in the sense that there is a locally finite, $N$-small chain, $z_{E}$, which represents a generator of $H_{n}(E, E-e)$ for each point $e \in E$.

Theorem 10.1 (Controlled Poincaré Duality Theorem) The morphism of graded objects in $\mathcal{A B}^{\mathcal{P} \mathcal{G}_{1}(E, p)}\left(\Sigma_{\mathcal{M}}^{-1}\right)$

$$
-\cap z_{E}: \Delta_{\mathcal{M}, N}^{*}\left((E, p)^{\sim}\right) \rightarrow \Delta_{n-*}^{\mathcal{M}, N}\left((E, p)^{\sim}\right),
$$

commutes with the coboundary/boundary morphisms, and the induced morphism

$$
-\cap\left[z_{E}\right]: H_{\mathcal{M}, N}^{*}\left((E, p)^{\sim}\right) \rightarrow H_{n-*}^{\mathcal{M}, N}\left((E, p)^{\sim}\right)
$$

is an isomorphism in the category $\mathcal{A B}^{\mathcal{P} \mathcal{G}_{1}(E, p)}\left(\Sigma_{\mathcal{M}}^{-1}\right)$.

\section{Proof :}

Since $z_{E}$ is a cycle, Proposition 9.1 shows that $-\cap z_{E}$ commutes with $\partial$ and $\delta$. Moreover, it also shows that the induced map on the (co)homology level depends only on the locally finite homology class $\left[z_{E}\right]$. To prove that it is an isomorphism, we shall use Proposition 3.3. Therefore, we have to describe a representing natural transformation. We can take this to be $C: H_{\mathcal{M}, N}^{q}(E, p) \rightarrow H_{n-q}^{\mathcal{M}, N}(E, p) \circ L$ where

$$
C_{(K, e)}=\left(-\cap q_{(K, e)}^{\#}\left(z_{E}\right)\right)_{*}: H_{c, N}^{q}\left(\left(p^{-1} L K, e\right)^{\sim}, \complement\left(p^{-1} K, e\right)^{-}\right) \rightarrow H_{n-q}^{L}\left(\left(p^{-1} L K, e\right)^{\sim}\right) .
$$

Two instances of this transformation fit into the following diagram, where the maps $\eta$ and $i$ are defined by requiring that they factor the natural map $\left(p^{-1} L K, e\right)^{\sim} \rightarrow\left(p^{-1} L^{2} K, e\right)^{\sim}$ as $\left(p^{-1} L K, e\right)^{\sim} \stackrel{\eta}{\longrightarrow}\left(p^{-1} L K, e\right)^{-} \stackrel{i}{\longrightarrow}\left(p^{-1} L^{2} K, e\right)^{\sim}$.

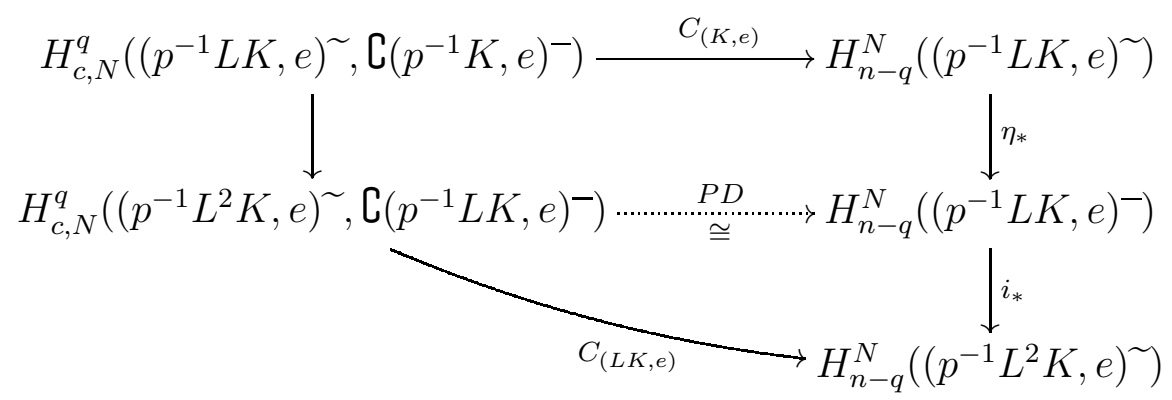

Naturality of $C$ viz-a-viz maps induced by the inclusion $p^{-1} L K \subseteq p^{-1} L^{2} K$ show commutativity of the outer diagram. Moreover, the left hand vertical map and the right hand vertical composition are instances of the transformations $\tau$ that appear in the criteria of Proposition 3.3. Therefore, if the dotted arrow can be filled in by an isomorphism, preserving commutativity, then commutativity of the rectangle suffices to show that the transformation $C$ is an eventual monomorphism, while commutativity of the triangle shows $C$ to be 
an eventual epimorphism. Altogether, then, Proposition 3.3 finishes the proof, modulo the existence of the dotted arrow.

In the argument for this, we shall use the abbreviation $V_{i}=\left(p^{-1} L^{i} K, e\right), i=0,1,2$, where $L^{0} K=K$. We can use the Poincaré Duality isomorphism of [6], Proposition 7.14, p. 297 to the triple $\complement V_{1}^{-} \subseteq V_{2}^{\sim}=V_{2}^{\sim}$ to obtain the isomorphism

$$
P D: \check{H}_{c}^{q}\left(V_{2}^{\sim}, \complement V_{1}^{-}\right) \rightarrow H_{n-q}\left(V_{1}^{-}\right)
$$

Since $V_{2}^{\sim}$ is a manifold, and therefore an ENR, [6], p. 289, shows that $\check{H}_{c}^{q}\left(V_{2}^{\sim}, \complement V_{1}^{-}\right)$ coincides with the bounded version, $\check{H}_{b}^{q}\left(V_{2}^{\sim}, \complement V_{1}^{-}\right)$, and by $(6.23)$ of the same page in [6], this means that $\check{H}_{c}^{q}\left(V_{2}^{\sim}, \complement V_{1}^{-}\right)$is the colimit of the ordinary Cech cohomology groups $\breve{H}^{q}\left(V_{2}^{\sim}, \complement C\right)$ as $C$ ranges over the directed set (under reverse inclusions) of all compact subsets $C \subseteq V_{1}^{-}$. Since $\left(V_{2}^{\sim}, \complement C\right)$ is a manifold pair, Proposition 6.23 of [6], p. 285 allows us to replace $\check{H}^{q}\left(V_{2}^{\sim}, \complement C\right)$ by $H^{q}\left(V_{2}^{\sim}, \complement C\right)$ in the colimit, and the upshot of all this is that $\check{H}_{c}^{q}\left(V_{2}^{\sim}, \complement V_{1}^{-}\right)$and $H_{c}^{q}\left(V_{2}^{\sim}, \complement V_{1}^{-}\right)$are identical. In addition to this "un-checking" we also note that the restriction to $N$-small chains throughout does no harm to the whole argument.

Altogether, we do have the dotted isomorphism. Proving that it makes the diagram commute is left to the reader. It is a slightly lengthy exercise in handling the various colimits that enter into Dold's definition.

\section{Appendix}

\subsection{Chain "groups" are projective}

Let $S=S_{k}^{N}$ be the set of all $N$-small singular $k$-simplices $\sigma: \Delta^{k} \rightarrow E$, and let $v_{0}$ be the initial vertex of $\Delta^{k}$. To each $\sigma$ we associate the object

$$
v(\sigma):=\left(K_{\sigma}, e_{\sigma}\right):=\left(\left\{p \sigma\left(v_{0}\right)\right\}, \sigma\left(v_{0}\right)\right) \in\left|\mathcal{P G}_{1}(E, p)\right| .
$$

In the terminology of [4] and [1], the pair $(S, v)$ is a basis for a free object $F=F(S, v)$ in $\mathcal{A B}^{\mathcal{P} \mathcal{G}_{1}(E, p)}\left(\Sigma_{\mathcal{M}}^{-1}\right)$. By definition, for any $(K, e), F(K, e)$ is the free abelian group generated by all pairs $(\sigma,(i, \omega))$, where $\sigma \in S$ and $(i, \omega):\left(K_{\sigma}, e_{\sigma}\right) \rightarrow(K, e)$ in $\mathcal{P} \mathcal{G}_{1}(E, p)$. For a morphism $(j, \rho):(K, e) \rightarrow\left(K^{\prime}, e^{\prime}\right)$, the induced homomorphism $F(K, e) \rightarrow F\left(K^{\prime}, e^{\prime}\right)$ maps the generator $(\sigma,(i, \omega))$ to the generator $(\sigma,(j, \rho) \circ(i, \sigma))$. The following proposition is easy to prove.

Proposition 11.1 Suppose given an object $G \in \mathcal{A B}_{1}^{\mathcal{P G}}(E, p)$, a monoid element $L$, and for each $\sigma \in S$ an element $g_{\sigma} \in G\left(L K_{\sigma}, e_{\sigma}\right)$. These data determine a unique natural transformation $g_{L}: F \rightarrow G \circ L$, for which $g_{L}\left(K_{\sigma}, e_{\sigma}\right): F\left(K_{\sigma}, e_{\sigma}\right) \rightarrow G \circ L\left(K_{\sigma}, e_{\sigma}\right)$ maps the generator $\left(\sigma, 1_{\left(K_{\sigma}, e_{\sigma}\right)}\right)$ to $g_{\sigma}$.

Of course, $g_{L}$ represents a morphism $g: F \rightarrow G$ in the category of fractions $\mathcal{A B}^{\mathcal{P G} \mathcal{G}_{1}(E, p)}\left(\Sigma_{\mathcal{M}}^{-1}\right)$, and it is not hard to see when two sets of data, say $\left(L,\left\{g_{\sigma}\right\}\right)$ and $\left(L^{\prime},\left\{g_{\sigma}^{\prime}\right\}\right)$, give identical 
morhisms $g=g^{\prime}: F \rightarrow G$, see Proposition 8.3 of [4] or Proposition 1.3, p. 126 of [1] for details.

We use this construction with $G=\Delta_{k}^{\mathcal{M}, N}\left((E, p)^{\sim}\right), L=N N^{-1}$ and $g_{\sigma}=\widetilde{\sigma}$, a lift of $\sigma: \Delta^{K} \rightarrow p^{-1} L K_{\sigma}$ to the covering space $\left(p^{-1} L K_{\sigma}, e_{\sigma}\right)^{\sim}$. Note that $\sigma$ does map into the component of $p^{-1}\left(L K_{\sigma}\right)$ containing $e_{\sigma}$, so such a lifting exists. There results a morphism $g: F(S, v) \rightarrow \Delta_{k}^{\mathcal{M}, N}\left((E, p)^{\sim}\right)$ with a representing natural transformation $g_{L}$ which is very concretely given. Therefore, it is not hard to check the conditions in (ii) and (iii) of Proposition 3.3, thereby establishing

Proposition 11.2 The above $g$ is an isomorphism $F\left(S_{k}^{N}, v\right) \cong \Delta_{k}^{\mathcal{M}, N}\left((E, p)^{\sim}\right)$ in the category of fractions $\mathcal{A B}^{\mathcal{P G}} \mathcal{G}_{1}(E, p)\left(\Sigma_{\mathcal{M}}^{-1}\right)$, i.e., the "group" of $N$-small singular $k$-chains on the universal covering $(E, p)^{\sim}$ is free with $\left(S_{k}^{N}, v\right)$ as a basis.

Proposition 11.1, together with the characterization of epimorphisms in Proposition 3.3, are also sufficient to prove the following proposition. The reader may consult [4] (Corollary 8.4 ) or [1] (Corollary 1.4, p. 126) for further details.

Proposition 11.3 $F(S, v)$ is a projective object in $\mathcal{A B}^{\mathcal{P G}} \mathcal{G}_{1}(E, p)\left(\Sigma_{\mathcal{M}}^{-1}\right)$.

\subsection{Remarks on Poincaré duality "downstairs"}

If we want to treat cochains and cohomology "downstairs", i.e., for the controlled space $(E, p)$ itself rather than its universal cover, we can take a slightly simpler setup. In fact, we may work with all singular chains rather than the $N$-small ones, and the $q$-th singular cochain "group" $\left(\in \mathcal{A B}^{\mathcal{P}}\left(\Sigma_{\mathcal{M}}^{-1}\right)\right)$ is simply the covariant functor

$$
\Delta_{\mathcal{M}}^{q}(E, p): K \mapsto \Delta^{q}\left(E, \complement\left(p^{-1} K\right)\right) .
$$

\subsection{Remarks on the cellular (or simplicial) case}

In our treatment of chains we worked in parallel with the singular and the cellular case, but from then on, we concentrated on the singualr case. However, the reader should have no great difficulties with an adaptation of the rest of our treatment to the cellular world. The key is the asumption that the cells are bounded by some monoid element so that the smallest subcomplex fragmentation is equivalent to the inverse image fragmentation, cf. section 5, especially Remark 5.2. Similarly, one could work in a simplicial setting throughout. 


\section{References}

[1] Anderson, D.R., Munkholm, H.J.: Boundedly Controlled Topology. Lecture Notes in Math. 1323, Springer-Verlag, Berlin - Heidelberg - New York 1988

[2] Anderson D.R., Munkholm, H.J.: Continouosly Controlled K-theory with Variable Coefficients. J. Pure Appl. Algebra 145 (2000), 215 - 266

[3] Attie O.: Quasi-isometry Classification of some Manifolds of Bounded Geometry. Math. Z. 216 (1994), $501-527$

[4] Christensen, R.d.: Elements of Monoidally Controlled Algebraic Topology and Simple Homotopy Theory. Ph.D. Thesis, IMADA, SDU, Odense, Denmark 2001

[5] Christensen, R.d, Munkholm, H.J.: Topology with Monoidal Control. Preprint, 2002, IMADA. SDU, Odense, Denmark. Submitted. Available from the K-theory Preprint Archives http://www.math.uiuc.edu/K-theory/0548

[6] Dold A.: Algebraic Topology. Springer-Verlag, Berlin - Heidelberg - New York 1972

[7] Greenberg M.J., Harper J.R.: Algebraic Topology. A First Course. Addison-Wesley Publishing Co. 1981

[8] Higson N., Pedersen E.K., Roe J.: $C^{*}$-algebras and Controlled Topology. K-theory 11 (1997), $203-239$

[9] Massey W.S.: Singular Homology Theory. Springer-Verlag, New York - Berlin - Heidelberg 1980

[10] Rotman J.J.: An Introduction to Algebraic Topology. Springer-Verlag, New York Berlin - Heidelberg 1988

[11] Schubert H.: Categories. Springer-Verlag, Berlin - Heidelberg 1972

[12] Vick J.W.: Homology Theory. Springer-Verlag, New York Inc. 1994

René dePont Christensen,

Dragebakken 354, DK 5250 Odense SV, Denmark

e-mail: rdp@dreamgate.dk

Hans J. Munkholm,

IMADA. University of Southern Denmark, Campusvej 55, DK 5230 Odense M, Denmark e-mail: hjm@imada.sdu.dk 